msh-mss Mathématiques et sciences humaines

145 | Printemps 1999

Géométrie et vision

\title{
Sur la configuration de Desargues
}

About the Desargues' configuration

\section{Marc Barbut}

\section{(2) OpenEdition}

\section{Journals}

Electronic version

URL: http://journals.openedition.org/msh/2811

DOI: $10.4000 / \mathrm{msh} .2811$

ISSN: 1950-6821

\section{Publisher}

Centre d'analyse et de mathématique sociales de l'EHESS

\section{Printed version}

Date of publication: 1 March 1999

ISSN: 0987-6936

\section{Electronic reference}

Marc Barbut, "Sur la configuration de Desargues », Mathématiques et sciences humaines [Online], 145 | Printemps 1999, Online since 10 February 2006, connection on 23 July 2020. URL : http:// journals.openedition.org/msh/2811; DOI : https://doi.org/10.4000/msh.2811 


\section{SUR LA CONFIGURATION DE DESARGUES}

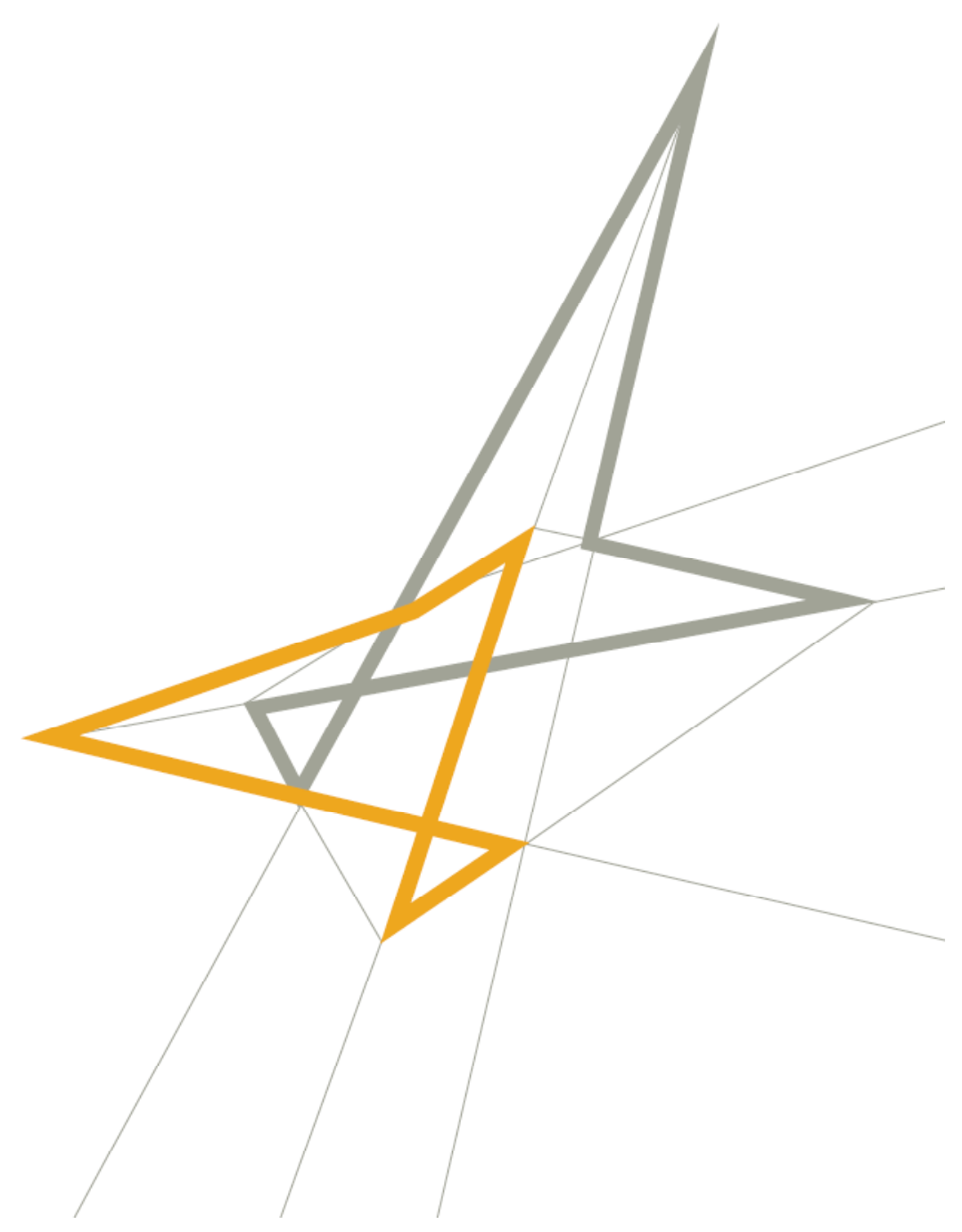

La figure ci-dessus représente la célèbre configuration arguesienne, du nom de son inventeur, le lyonnais Girard Desargues (1593-1662), père de la géométrie projective.

Cette configuration a été choisie comme emblème (on dirait aujourd'hui comme "logo") de notre revue dès sa création.

En elle se rencontrent en effet quatre domaines majeurs des mathématiques, pures ou appliquées, qui tous comptent beaucoup dans les rapports de celles-ci avec les sciences de l'homme : Géométrie, Combinatoire, Statistique, Algèbre.

On y reviendra dans un numéro ultérieur. Voici, dès maintenant, quelques indications.

GÉOMÉTRIE - G. Desargues l'a démontré : si, dans le plan ou dans l'espace euclidiens, deux triangles se déduisent l'un de l'autre par perspective (de centre A, par exemple) leurs 
côtés homologues se coupent deux à deux en trois points d'une même droite, l'axe de perspective associé (la droite a dans l'exemple).

De même de B et b; il y a ainsi dix couples de triangles perspectifs, chacun des dix points étant centre, et ayant un axe correspondant ; trouvez les huit autres.

Mais il y a aussi des couples de pentagones (non convexes) tels que chaque sommet du premier appartienne à un et un seul côté du second, et réciproquement.

D'ailleurs, la dualité point-droite de la géométrie projective plane est partout présente dans cette configuration.

COMBINATOIRE - Dix points, dix droites : par chaque point, il passe trois droites ; et chaque droite contient trois points. Remplaçons "points" par "trucs" et "droites" par "machins" : voici l'un de ces dispositifs réguliers que la Combinatoire affectionne. Identifions en outre chaque "truc" à son "machin" : nous obtenons ci-contre, un graphe régulier célèbre pour ses pentagrammes.

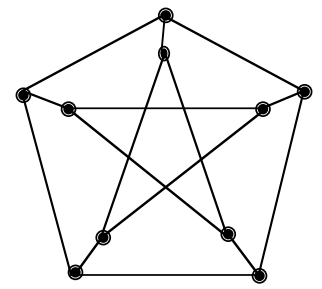

STATISTIQUE - Trucs et machins : si, comme pour le grand statisticien Ronald Fisher, ce sont "espèces" et "variétés" (par exemple diverses espèces de patates soumises à plusieurs variétés de traitement par des engrais), voici un bel exemple de ces plans d'expérience en blocs incomplets équilibrés dont il fut l'inventeur, et qui sont à la base de l'analyse de la variance.

ALGÈBRE, enfin - Envoyons la droite a à l'infini. Voici deux triangles homothétiques (par rapport au centre A). Envoyons de surcroît le point A lui-même à l'infini : les deux triangles se déduisent l'un de l'autre par translation.

Translation, homothétie : addition, multiplication (par un scalaire) ; les deux opérations au moyen desquelles sont définis les corps de nombres.

Et de fait, pour qu'une géométrie projective plane puisse être traitée analytiquement, et donc algébriquement, à la manière cartésienne, avec des coordonnées homogènes à valeurs dans un corps (fini ou non), il faut et il suffit que la configuration de Desargues y soit valide.

Dans l'espace de dimension 3 ou plus, le théorème de Desargues sur les triangles perspectifs est trivialement vérifié ; il l'est donc dans le plan, si ce dernier est plongé dans un espace de dimension 3.

Mais si la dimension de l'espace n'est que 2 ? Alors, il y a des géométries projectives non-arguesiennes, auxquelles correspondent des structures algébriques un peu baroques, et de maniement moins aisé que celle de corps. calcul.

Ainsi, la configuration de Desargues est intrinsèquement liée à nos habitudes de M. B. 